\title{
Perception of scenes in different sensory modalities: A result of modal completion
}

\section{Authors: Ronald P. Gruber \& Richard A. Block}

This is a postprint of an article that originally appeared in American Journal of Psychology in March 2017.

Gruber, Ronald P. , and Richard A. Block. "Perception of scenes in different sensory modalities: A result of modal completion ." The American Journal of Psychology 130, no. 1 (June 2017): 23-34. DOI: 10.5406/amerjpsyc.130.1.0023

Made available through Montana State University's $\underline{\text { ScholarWorks }}$ 


\title{
Perception of Scenes in Different Sensory Modalities: A Result of Modal Completion
}

\author{
RONALD P. GRUBER \\ Stanford University Medical Center \\ RICHARD A. BLOCK \\ Montana State University
}

Dynamic perception includes amodal and modal completion, along with apparent movement. It fills temporal gaps for single objects. In 2 experiments, using 6 stimulus presentation conditions involving 3 sensory modalities, participants experienced 8-10 sequential stimuli (200 ms each) with interstimulus intervals (ISIs) of $0.25-7.0 \mathrm{~s}$. Experiments focused on spatiotemporal completion (walking), featural completion (object changing), auditory completion (falling bomb), and haptic changes (insect crawling). After each trial, participants judged whether they experienced the process of "happening" or whether they simply knew that the process must have occurred. The phenomenon was frequency independent, being reported at short ISIs but not at long ISIs. The phenomenon involves dynamic modal completion and possibly also conceptual processes.

KEYWORDS: perception, multimodal perception, attention, modal completion, amodal completion, dynamic change, apparent movement, perceptual completion

A recent renaissance in the study of temporal illusions suggests that temporal processing occurs at many levels, involving such variables as temporal frequency, interstimulus intervals (ISIs), and durations. Examples include perceiving the world in slow motion during a car accident and the "stopped clock" illusion, in which the second hand of a clock sometimes seems to be stopped in place momentarily before it continues to tick at a normal pace (Yarrow, Haggard, Heal, Brown, \& Rothwell, 2001). In the case of ISIs, temporal processing effects are reported at many different time intervals, such as $20-30 \mathrm{~ms}$, 50-200 ms, 3,000 ms, and even longer (Block, 1979; Eagleman, 2008; Grondin, 2010).
For more than 125 years, American fournal of Psychology has published landmark articles on the psychology of time (Hancock, 2010, 2011; Hancock \& Block, 2012; Nichols, 1891; Zakay, 2007), but no researchers have reported evidence on the flow of time, in particular its dynamics. In this article, we report such evidence and introduce perceptual completion as an important factor in the evocation of that phenomenon.

Modal and amodal perceptual completion are essential for perceptual completion of a robust perception of the world (Kellman \& Shipley, 1992a, 1992b). Others have discussed it in great detail (Lin \& $\mathrm{He}$, 2012; Pessoa \& DeWeerd, 2003; Wokke, Vanden- 
broucke, Scholte, \& Lamme, 2013). It is often referred to as bridging the gap (Hubbard, 2006) or filling the gap (Macknik \& Martinez-Conde, 2014). Also, Clark (2002) noted that the brain processes more than is actually in external stimuli. Amodal perception is the perception of the whole of a physical structure when only parts of it affect the sensory receptors. The illusory perception is behind the occluder. An often-quoted example is the "dog behind a picket fence." Modal completion is a similar phenomenon in which a shape is perceived to be occluding other shapes even when that shape itself is not continuous or complete. An example is the illusory Kanizsa triangle, which appears to be occluding three disks and an actual outlined triangle. The illusory perception is in front of that which it is occluding (Singh, 2004). For a demonstration see Bach (2014). Some agree that much of what we experience in this world is an illusion (Ballard, 2002; Blackmore, Brelstaff, Nelson, \& Troscianko, 1995). Moreover, the illusory percepts that do fill the gap are most vital for dynamic as well as static perceptual completion.

Palmer, Kellman, and Shipley (2006) proposed a theory of spatiotemporal reliability that describes the visual information and processes that allow visible fragments to be revealed at different times and places because of motion and occlusion. They confirmed it with experiments using dynamic and static objects due to motion and occlusion, to be assembled into unitary perceived objects: A dynamic object is one that moves relative to a partial occluder, whereas a static (nondynamic) object is one that exhibits no motion, with the partial occluder doing the moving. For example, if an observer is on a fire truck viewing a parked car through a window that partly occludes vision, the car would be said to be a static object. Clearly, a dynamic representation of the elements of the car is necessary to allow recovery of it. The authors postulated a mental representation, a dynamic visual image, which briefly maintains shapes and updates positions of occluded fragments to connect them with visible regions. The idea that a representation of a complete object can be constructed through a series of separate fragmentary views is referred to as arthroscopic perception (Rock, 1997).

In the case of single objects, the phenomena of apparent movement and dynamic change are important parts of dynamic perceptual completion. To be sure, there is a difference between perceptual comple- tion for a static scene and a dynamic change between two stimuli. The former might involve a boundary extension, and the latter may involve representation momentum or representational gravity. However, the experimental phenomenon of filling the gap in the observer's world is strikingly similar, enough so that we may discuss perceptual completion in terms of its dynamic and adynamic components. Under the general heading of change detection (Flombaum \& Scholl, 2006; Kornmeier \& Bach, 2009; Simons, Mitroff, Franconeri, \& Peterson, 2003), dynamic change is defined as seeing the change in progress. It is perception of the transformation itself (see Rensink, 2002).

Hollingworth (2008) provided examples of dynamic change using a flicker paradigm for a featural change of a single object. A typical change was the replacement of one brief presentation (250-ms duration) of an object (e.g., a cup) by a slightly different object (e.g., a different type of cup), also of 250ms duration, with a blank ISI varying from 200 to $5,000 \mathrm{~ms}$. Participants reported a strong impression of "seeing the change occur" at $200 \mathrm{~ms}$, a weaker impression at 1,000 ms, and no impression of change at 5,000 ms. Nakashima and Yokosawa (2012) used a flicker paradigm to demonstrate dynamic change in a spatiotemporal situation. Their study used a brief visual stimulus of a bed (duration of 100-200 ms) followed by one that was identical, with the exception that its orientation was rotated $90^{\circ}$, with a black ISI of 100-2,000 ms in between. Participants usually reported that they could "see the change occur" if the ISI was less than 1,000 ms. However, no mention was made as to whether the participants' perceptual experience involved actual motion of the bed. Apparent motion is typically not seen above ISIs of $500 \mathrm{~ms}$; thus, the experience of their participants could easily have been motion at the shorter ISIs and more likely something else that we will be discussing.

We previously reported a similar finding for the temporal gaps of scenes, not just single objects (Bach, 2015; Block \& Gruber, 2014; Gruber \& Block, 2013). In a spatial change situation we used 10 frames (snapshots) captured from a man-walking scene to be representative of the walk from its beginning to its end. ISIs were $0.5,3.0$, and $7.0 \mathrm{~s}$ and were consistent across trials. To evaluate the experiential phenomenon that occurs in a scene, not just a single object, we chose a dichotomous forced choice for the partici- 
pants, hoping to elicit the complete dynamic experience. The choice questions for the walking man were "Yes, I could see it happening. I sensed his motion across the parking lot" or "No, I could not see it actually happening or sense his motion. But the individual slides told me he must have gone from one side to the other." At short ISIs the answers were uniformly yes, but most answered $n o$ at very long ISIs $(7 \mathrm{~s})$.

In a featural (color) change situation, 10 frames captured from toasting bread were used. The choices were "Yes, I could see it actually happening. I saw it become toasted" or "No, I could not see it actually happening. But the individual slides told me that it must have happened." Here, too, participants tended to respond yes for the short ISIs and no as the ISI was lengthened. Unlike the results of the dynamic change studies, the phenomenon is slightly different. For one, it was elicited at long ISIs closer to $3 \mathrm{~s}$, suggesting that the perceptual completion for scenes may not be exactly the same as that (dynamic change) for single objects. Moreover, it could not be established if the experiential phenomenon was perceptual or conceptual (represented but not experienced). Viewing dynamic processes as solely perceptual or solely conceptual may be oversimplified and potentially misleading (Hubbard, 2005, 2006, 2014). Those results prompted our present study to examine other aspects of the experiential phenomenon (presumably modal completion) noted in spatial and featural scenes of the visual modalities and also to determine whether it exists in other sensory modalities.

\section{EXPERIMENT 1}

We conducted two experiments, each with six repeated-measures (within-subject) conditions. We first report methods for Experiment 1, then how they differed for Experiment 2.

\section{METHOD}

Participants were four women and two men, ages 29-64 years $(M=44)$. They volunteered to participate in an institutional review board-approved study and were naive with respect to the purpose of the research. The experiments were conducted in multiple sessions. They were seated for video presentation and sat in front of a laptop computer at a distance of approximately $50 \mathrm{~cm}$ and were told they would see videos, hear audio tracks, or experience tactile stimulation. The $8-10$ stimuli were presented in a random order and displayed in a visual, auditory, or tactile modality, with fixed ISIs between them typically ranging from 0.5 to $7.0 \mathrm{~s}$. The form of each of the 8-10 stimuli was consistent within a trial (i.e., all the stimuli in a given trial were visual, all were auditory, or all were tactile).

Participants were then immediately given a forcedchoice dichotomous (binary) question on what they had experienced: They were asked to report that either they experienced something happening, such as walking, movement, or object change, or they did not experience anything happening except for a series of stimuli. The reason that the word happening was chosen is for two reasons: We needed to find a word that conveyed a nonspecific experiential phenomenon of an action-filled scene, and in some flow-of-time discussions (Rickles \& Kon, 2014), the term happening is occasionally used to describe the dynamic quality of the flow of time. The chances of confusing happening with apparent movement were not considered to be an issue at ISIs $>0.5 \mathrm{~s}$, at which apparent movement tends not to occur. Finally, participants were told that there were no correct or incorrect answers. Participants were exposed to six conditions:

\section{Condition 1. Spatiotemporal Change}

With Visual Obstruction (Fence)

Three videos were made of eight slides each. Each slide had nine parallel white picket fences $(4$ feet high, 7 feet wide), each separated by 4 feet. In each slide a walking man was standing in front of a different fence. The man was placed such that he appeared to be going from right to left and from one fence to the next. In each slide his path was apparently obstructed by the fence in front of him. The slide duration was $200 \mathrm{~ms}$. The black ISI for each of the three videos was $0.5,3.0$, or 7.0 s. After each video the participant was asked to make a binary-choice (dichotomous) decision, choosing either "Yes, I could see it happening. I sensed his motion from one side to the other despite the fences" or "No, I could not actually see it happening. I saw the man in front of one fence after another." The scene is shown in Figure 1.

\section{Condition 2. Featural Change: Multiple Objects (Center)}

Three videos were made of 10 slides each. Each slide had 1 of 10 different objects (e.g., camera, car, guitar) centrally located on the computer screen (Figure 2). As each slide was presented, a different object would suddenly appear in the same central location. The intent was to notice whether the video evoked an experience of happening. Again, the ob- 


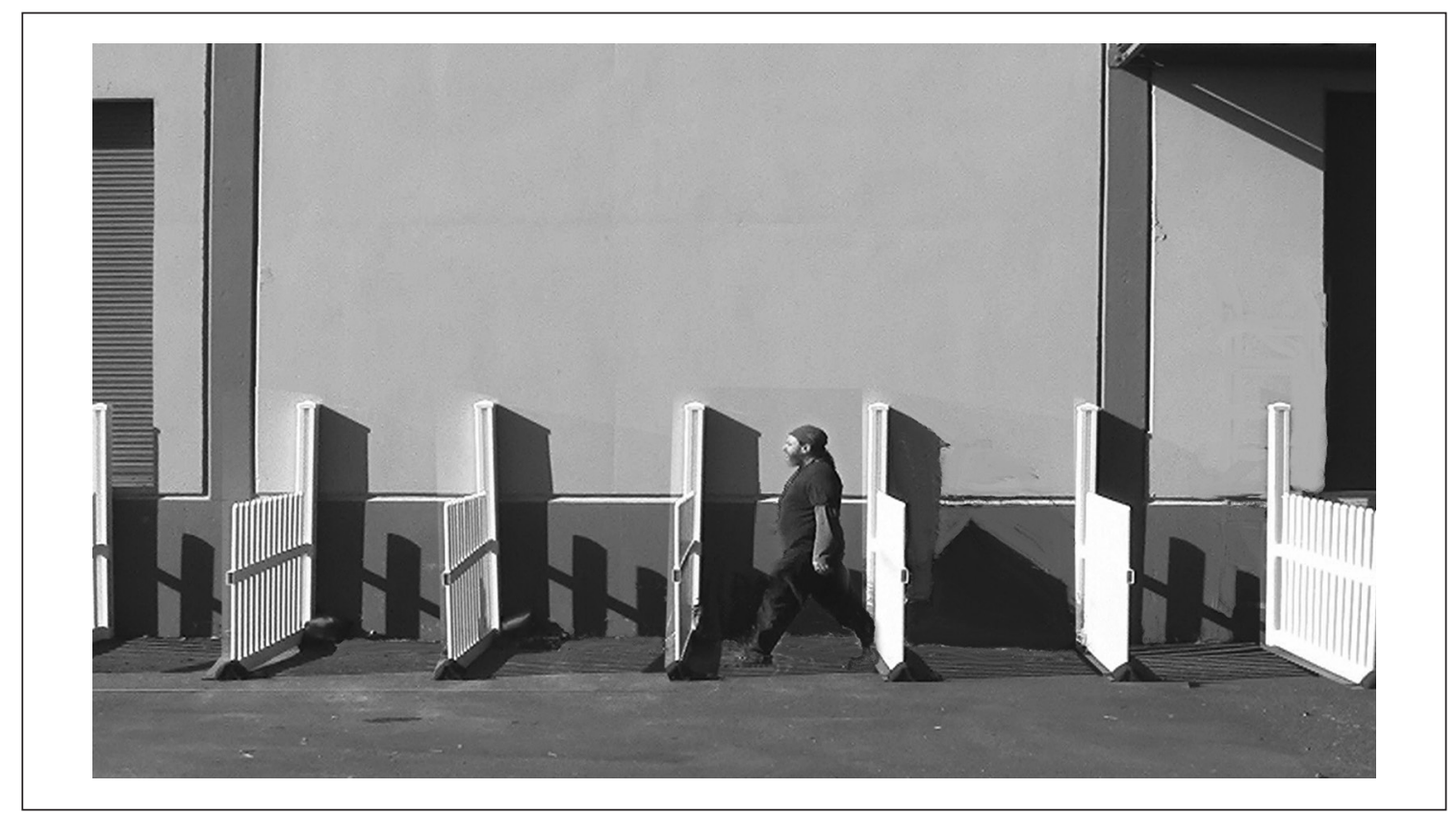

FIGURE 1. Scene of man walking "through" the fences

ject duration was $200 \mathrm{~ms}$ and the black ISI was 0.5 , 3.0, or 7.0 s. Participants then made the same binary decision: "Yes, I could sense something happening but do not know what" or "No, I could not sense anything happening. It was just a series of different objects."

\section{Condition 3. Spatiotemporal and Featural Change: Multiple Objects (Right-to-Left)}

Three videos were made of 10 slides each. Each slide had one of 10 different objects (see Figure 2), now located at a different spatial positions from right to left. Thus, as each object was presented a different object would soon appear at a different position just to the left of where the previous object was seen. The intent was to determine whether participants would experience something happening between stimuli (objects). The black background remained station-

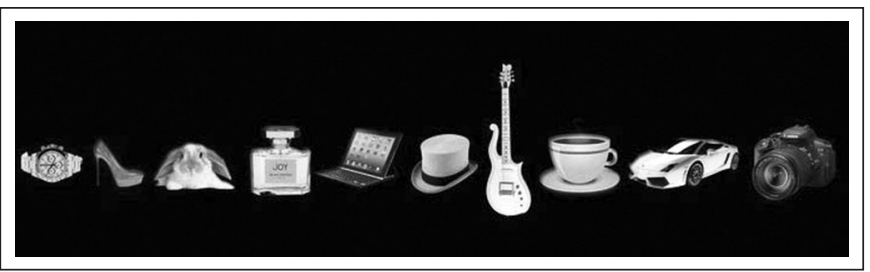

FIGURE 2. Stimuli presented in Conditions 2 and 3, one at a time ary despite the change in target identity. Conceivably, the change in target identity could be a cause of disrupting dynamic processing such as representational momentum (Kelly \& Freyd, 1987). However, no displacement of the stimulus and no apparent movement were anticipated at these long ISIs $\left(0.5^{-7} \mathrm{~s}\right)$. It remained to be seen whether the modal perception of happening would be disrupted.

As in Condition 1, the slide duration was $200 \mathrm{~ms}$ and the black ISI was $0.5,3.0$, or $7.0 \mathrm{~s}$. After each video the participants was asked to make a binary decision, either "Yes, I could sense something happening or moving from one side to the other" or "No, I could not sense something actually happening or moving from one side to the other."

\section{Condition 4. Attention Shift (for Walking Man)}

Three videos of a walking man scene identical to those in Block and Gruber (2014) were made. Each showed 10 slides of a man walking across a parking lot at a different location from right to left. Slide durations and ISIs were as before. The scene occupied $75 \%$ of the computer screen and was randomly shifted to the four corners. This required the observer to shift attention each time a new stimulus appeared. The shift design was similar to that of Nakashima and Yokosama (2012). Participants then made the decision: "Yes, I could see it happening. I sensed 
his motion across the parking lot" or "No, I could not see it actually happening or sense his motion. But the individual slides told me he must have gone from one side to the other."

\section{Condition 5. Auditory Stimulus (Bomb Drop)}

A 6-s audio bomb drop recording consisted of a continuously decreasing frequency. It was an artificially created bomb drop (Lg, n.d.). Ten serial equally spaced segments (200 $\mathrm{ms}$ ) of the bomb drop were extracted. The peak frequencies declined from approximately $13 \mathrm{kHz}$ to $12 \mathrm{kHz}$. The recording was played from a Dell laptop at a distance of $50 \mathrm{~cm}$ from the participant and a measured 68.4-74.2 dB loudness at the peaks. Three audio tracks were made consisting of the 10 audio segments of $200 \mathrm{~ms}$ with silent ISIs of each of the three tracks being either $0.5,3.0$, or $7.0 \mathrm{~s}$. Thus, the setup was similar to that of the nonaudio experiments. Moreover, the audio properties were kept constant throughout for all participants and all audio exposures. The only thing differentiating the three tracks was the ISI.

Participants then made the decision: "I could hear the bomb drop. I could hear it happen" or "I could only hear individual sounds from a bomb dropping. Audio tracks were randomly presented."

\section{Condition 6. Haptic Stimulus (Forearm Bug)}

Participants were shown a 1-inch artificial orange plastic bug with polka dots and told that it would be applied gently to the forearm. First a 10-inch masking tape (divided into 10 segments) was applied to the forearm as it was outstretched on the table (Figure 3). The participant was asked to close his or her eyes and turn his or her head to the side. There was the possibility that a participant might see his or her reflection in a nearby window. However, the lids appeared completely closed to the experimenter at all times. To provide a sensation of crawling up the forearm, a device was created from a glucose finger stick. The needle of it was bent at a right angle so that there would be no skin penetration. Gentle pressure by the experimenter to the device released the blunted needle, which in turn provided a sensation of pointed touch similar to a pencil point. It was not practical to simulate a bug by having multiple needles to represent the multiple legs of a bug. Finally, because multiple points were not used, the two-point discrimination of the forearm was irrelevant.

Using a stopwatch, the experimenter applied the device to the area adjacent to the 10 marks on the tape in successive order. In other words, the bug was "walked up the forearm." Participants then made the decision: "I sensed the insect crawling up my forearm. I sensed it happening" or "I did not sense it actually crawling up my forearm. However, I did feel it multiple times."

\section{EXPERIMENT 2}

\section{METHOD}

To validate the results of Experiment 1, it was repeated with a protocol improvement that would remove any ambiguity of the results. Participants were 10 women, ages $18-60$ years $(M=41)$. The same six stimulus conditions as in Experiment 1 were used. However, instead of being asked for a binary decision as in Experiment 1, participants were asked after each stimulus sequence to make a rating on a 5-point scale, with $1=$ very well, $2=$ fairly well, $3=$ somewhat, $4=$ a little, and $5=$ not at all. The words see and sense were not used as in Experiment 1. We also removed the term motion from the questions. Thus, the participants' responses were more likely to be confined to the experiential phenomenon of happening.

As before, the term happening was used because it seemed to be the best way for the participant to be able to express the feeling of a nonspecific, actionfilled scene of events. Because the objects were so qualitatively different from one another, there was no concern that the participant could morph one into the other, as in the Hollingworth (2008) study. Also, an issue of apparent movement (phi) was not anticipated because the ISIs were greater than $0.5 \mathrm{~s}$, which is commonly the largest interval at which apparent movement occurs.

\section{RESULTS}

In Experiment 1, data were analyzed by using Cochran's $Q$ test and accompanying standard errors (SEs) for this dichotomous response method design (My-

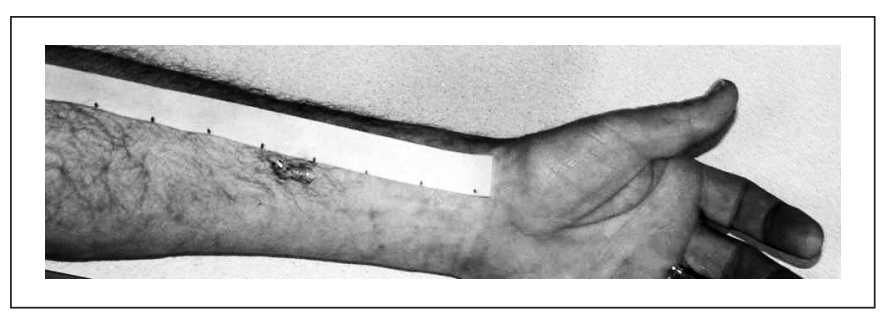

FIGURE 3. Artificial bug "crawling" up the forearm 
ers, Well, \& Lorch, 2010). SEs were calculated, as recommended, by taking the square root of the quantity $(p *(1-p) / n$, where $p$ is the proportion of "yes" responses, $1-p$ is the proportion of "no" responses, and $n=6$. We show proportion report and $S E \mathrm{~s}$ in Figures 4-9.

In Experiment 2, data were analyzed using repeated-measures ANOVAs testing for linear and quadratic trends (see Keppel \& Wickens, 2004), the typical analysis for our design using an interval scale (dependent variable). We show mean rating and SEs in Figures 4-9.

\section{Condition 1: Spatiotemporal Change}

With Visual Occlusion (Fence)

Recall that in Condition 1, participants viewed sequential images with visual occlusion (see Figure 1). Figure 4 shows the proportion of participants reporting that happening decreased across ISIs. In Experiment 1, Cochran's $Q$ test revealed a significant difference between ISIs, $\chi^{2}(2)=8.40, p=.015$. In Experiment 2, only the linearly decreasing trend was significant, $F(1,9)=51.3, p<.001$. The participants were not specifically asked whether they saw the man passing through or around the fence. However, anecdotally, some offered their observation that the man appeared to go through the fence.

Assuming that part of the experimental phenomenon at the $0.5^{-\mathrm{s}}$ ISI was apparent movement, categorizing it in terms of long-range movement or short-range movement would be of little help. A long-range motion system is responsible for classic phenomena of apparent motion with large-scale, individual figures. It is thought to occur much later in visual processing, after information from the two eyes has been integrated. Short-range motion system is responsible for performance on a random dot kinematogram. It is thought to occur fairly early in visual processing, before information from the two eyes has been integrated and before shape and color have been extensively analyzed.

Condition 2: Featural Change: Multiple Objects (Centered) Recall that in Condition 2, participants viewed sequential images centered, with visual occlusion (see Figure 2). Figure 5 shows the proportion of participants reporting that happening decreased across ISIs. Cochran's $Q$ test revealed a significant difference between ISIs, $\chi^{2}(2)=6.50, p=.04$. Thus, even when a series of various stimuli were presented, responses still revealed judgments of happening. As in Condition 1, with an increase of ISI, the proportion of participants reporting happening decreased. In Experiment 2, only the linearly decreasing trend was significant, $F(1,9)=14.5, p=.004$.

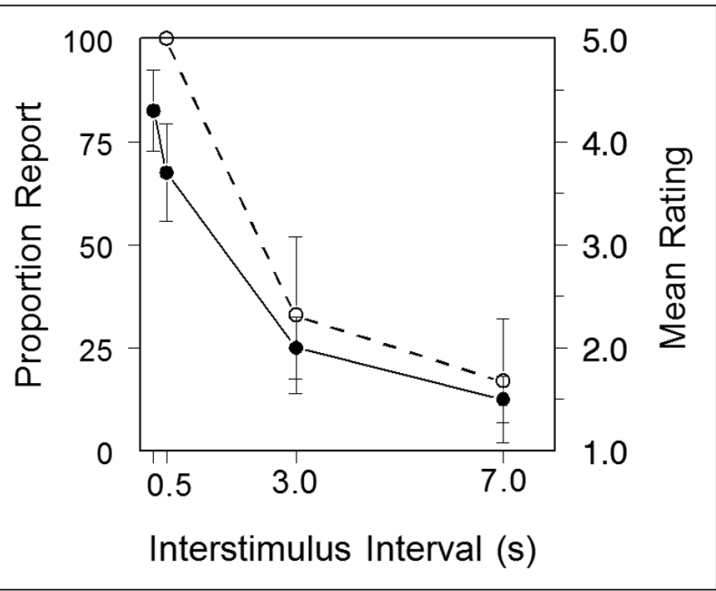

FIGURE 4. Proportion report in Condition 1 of Experiment 1 (left $y$-axis, unfilled circles) and mean rating as a function of interstimulus interval (s) in Condition 1 of Experiment 2 (right $y$-axis, filled circles). SE bars are shown for each data point

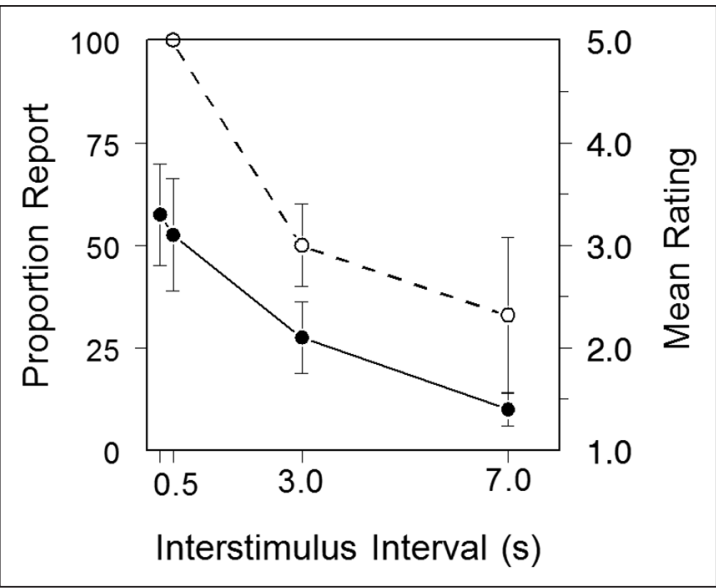

FIGURE 5. Proportion report in Condition 2 of Experiment 1 (left $y$-axis, unfilled circles) and mean rating as a function of ISI (s) in Condition 2 of Experiment 2 (right $y$-axis, filled circles). SE bars are shown for each data point 
Condition 3. Spatiotemporal and Featural Change: Multiple Objects (Right-to-Left)

Recall that in Condition 3, participants viewed multiple images presented from right to left on the screen (see Figure 2). As shown in Figure 6, the proportion of participants reporting happening decreased across ISIs. Cochran's $Q$ test revealed a significant difference between ISIs, $\chi^{2}(2)=6.00, p=.05$, and the only linearly decreasing trend was significant in Experiment $2, F(1,9)=13.7, p=.005$.

The effect was slightly weaker than in Condition 2, and we are not entirely sure why that should be. However, the reason we chose to look for the phenomenon of happening in a featural-only situation is to be sure that apparent movement can be ruled out as a cause of happening. If both apparent movement and happening were contributing factors, a weaker response would be expected in a featuralonly situation. Finally, we also note that whereas in Condition 2 the stimuli were sequentially presented at the same location, the stimuli in Condition 3 were sequentially presented at different (adjacent) locations. It raises the question as to whether object location in Condition 3 is a potentially significant factor. Testing for that possibility would have required randomizing the order of the objects and many more test groups. That was not considered a reasonable concern.

\section{Condition 4. Attention Shift (for Walking Man)}

Recall that in Condition 4, participants viewed multiple images presented from right to left on the screen (see Figure 2). As shown in Figure 7, the proportion of participants reporting happening decreased across ISIs. Cochran's $Q$ test revealed a significant difference between ISIs, $\chi^{2}(2)=6.00, p=.05$, and the linearly decreasing trend was significant in Experiment 2, $F(1,9)=110.2, p<.001$, as was also the quadratic trend, $F(1,9)=26.1, p=.001$.

\section{Condition 5. Auditory Stimulus (Bomb Drop)}

Recall that in Condition 5, participants heard audio tracks of a bomb dropping. As shown in Figure 8, the proportion of participants reporting happening decreased across ISIs. Afterwards, some of the participants offered their opinion that the longer ISI exposures did not sound like a bomb. However, not experiencing happening should not be interpreted to mean that the participant did not know that the sounds represented a bomb drop. That is because participants were exposed to multiple tracks of a bomb drop, some at short (0.5 s) ISIs that clearly

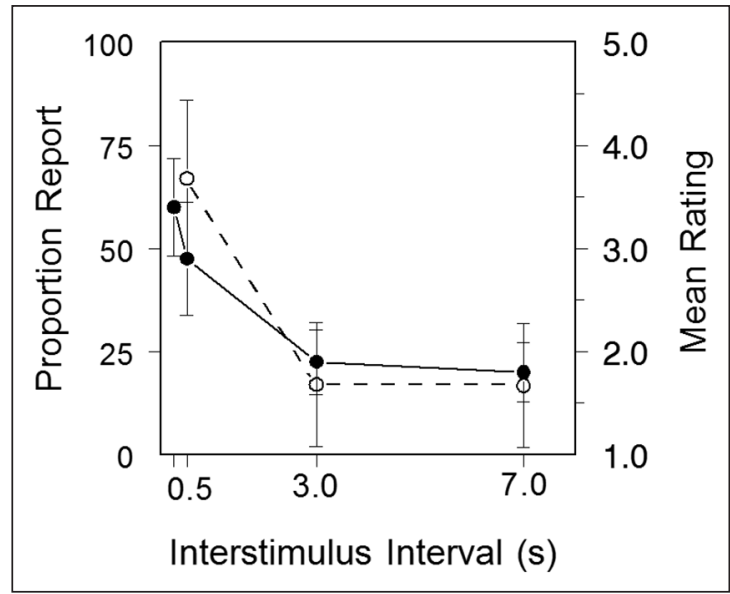

FIGURE 6. Proportion report in Condition 3 of Experiment 1 (left $y$-axis, unfilled circles) and mean rating as a function of ISI (s) in Condition 3 of Experiment 2 (right $y$-axis, filled circles). SE bars are shown for each data point

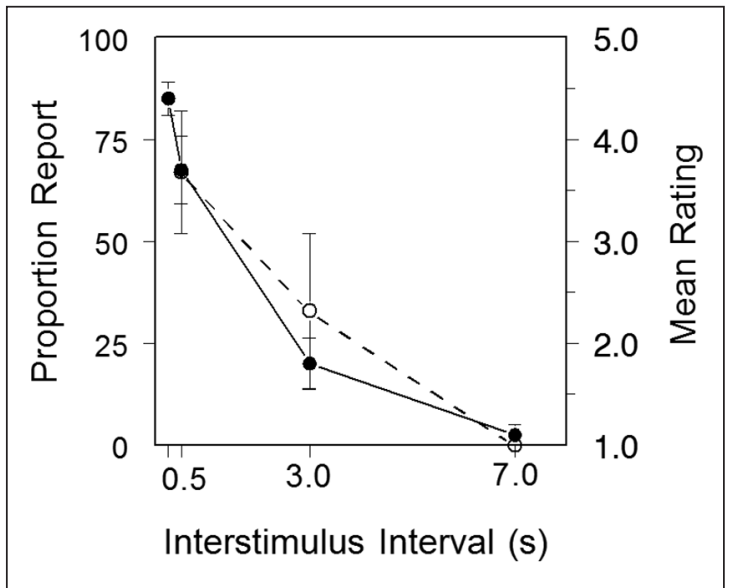

FIGURE 7. Proportion report in Condition 4 of Experiment 1 (left $y$-axis, unfilled circles) and mean rating as a function of ISI (s) in Condition 4 of Experiment 2 (right $y$-axis, filled circles). SE bars are shown for each data point 


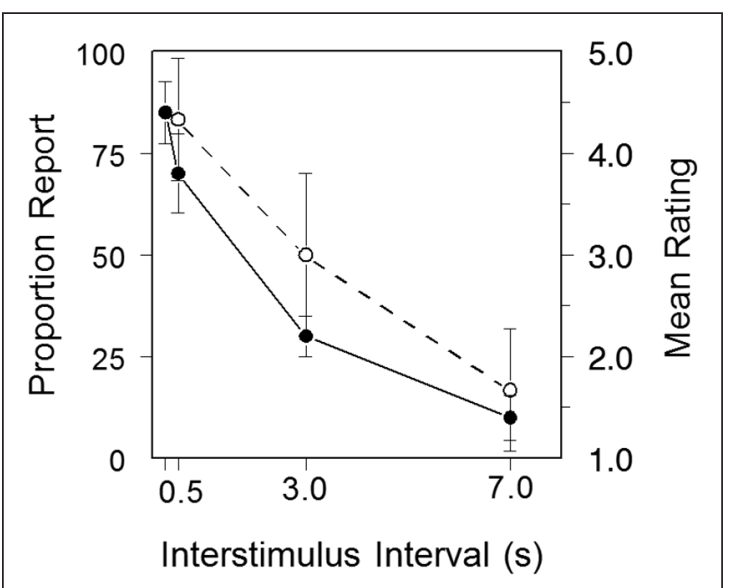

FIGURE 8. Proportion report in Condition 5 of Experiment 1 (left $y$-axis, unfilled circles) and mean rating as a function of ISI (s) in Condition 5 of Experiment 2 (right $y$-axis, filled circles). SE bars are shown for each data point

sound like a bomb drop. Apparently, however, the widely spaced sounds (ISIs of $7 \mathrm{~s}$ ) simply did not convey the perceptual experience of a bomb drop happening even if the participant knew that those sounds must be the from a bomb drop.

Cochran's $Q$ test revealed a significant difference between ISIs, $\chi^{2}(2)=6.00, p=.050$, and the linearly decreasing trend was significant in Experiment 2, $F(1,9)=69.3, p<.001$, as was the quadratic trend, $F(1,9)=45.6, p<.001$.

\section{Condition 6. Haptic Stimulus (Forearm Bug)}

A crawling sensation of the bug moving up the forearm as opposed to the bug touching the forearm multiple times was experienced by all participants at low (0.5-s) ISIs, although it decreased at medium and long ISIs. The general qualitative nature of this haptic response was similar to the auditory response in Condition 5 , as well as those in all previous conditions.

Recall that in Condition 6, participants experienced a haptic stimulus (forearm bug). As shown in Figure 9, the proportion of participants reporting happening decreased across ISIs. Cochran's $Q$ test revealed a significant difference between ISIs, $\chi^{2}(2)=$ $8.40, p=.015$, and only the linearly decreasing trend was significant in Experiment 2, $F(1,9)=19.9, p=$ .002 .

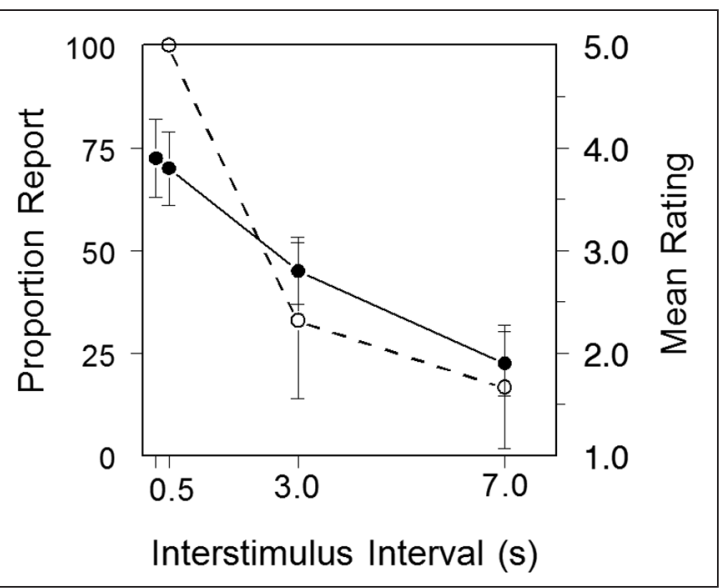

FIGURE 9. Proportion report in Condition 6 of Experiment 1 (left $y$-axis, unfilled circles) and mean rating as a function of ISI (s) in Condition 6 of Experiment 2 (right $y$-axis, filled circles). SE bars are shown for each data point

\section{DISCUSSION}

Dynamic Change Versus Percept Versus Modal Completion The experiments in this study were somewhat similar to the dynamic change studies (Hollingworth, 2008; Nakashima \& Yokosawa, 2012) but not the same in important ways. By using only two stimuli, Hollingworth's experiment could test for a specific featural change (from one type of cup to another). Similarly, Nakashima and Yokosawa's experiment tested for a specific spatial (rotational) change. However, two stimuli may not be enough to acquire a dynamic experience (happening) of a more complex process such as falling (bomb) or crawling (bug). A different explanation is needed for the multiple-objects experiments. It could be that there is a subthreshold experience of happening for any two stimuli of long ISIs. But when multiple such stimuli are presented there is an additive effect that would not occur for just two stimuli.

In the case of the multiple-objects experiments (Conditions 2 and 3 ) we were again looking for a new experiential phenomenon not previously described. It is the perception that one could also refer to as an experience of action filled. We described it previously in toasting experiments (Gruber, Bach, \& Block, 2015) in which the process of toasting (in a 
series of slides separated by ISIs ranging from 0.5 to 7 s) was experienced by participants at all intervals but mostly at short ones. The term modal completion (as opposed to percept) is a reasonable choice to describe happening because it is an illusory phenomenon that is superimposed on other perceptions. Modal completion is also viewed as filling the gap. In that sense, happening does fill the gap if one assumes that all visual perception is discrete (VanRullen $\&$ Koch, 2003). And the evidence for that is quite strong; it is, in fact, approximately $10 \mathrm{~Hz}$. Therefore, the net result is that happening is a major contributor to the dynamic flow of time. Motion is the other major contributor.

We tested three sensory modalities (visual, auditory, and haptic). To elicit any perceptual completion between stimuli that had a dynamic quality to it, we used a forced-choice question (Experiment 1 ). We used the term happening because it implied an action-filled experience. The expression "a change occurs" for haptic and auditory scenes seemed too ambiguous. In the second part of the forced choice we were able to be specific. We allowed participants to have the option of stating that all he or she experienced was a series of stimuli. By being specific, such as asking whether the falling of a bomb or crawling up the forearm was happening, we could be fairly certain that the participant was experiencing a dynamic phenomenon. Of course, we are assuming that the dynamic experience is the result of a filling in of the gap between the stimuli. Many types of dynamic experiences do not involve filling in of a gap per se but rather involve an extension in a specific direction away from the stimulus; for example, representational momentum involves a dynamic forward in the direction of motion, and boundary extension involves a dynamic outward beyond the initial view (Hubbard, 2005).

Temporal gaps are probably not densely filled by happening any more than is the case for amodal completion during the tunnel effect (Burke, 1952; Michotte, Thines, \& Crabbe, 1991). In the tunnel effect, some observers have a weak but distinct impression of seeing an object move through the tunnel (Burke, 1952). In our experiments, it is possible that the participants had a weak impression of actual bug crawling between forearm stimulations and an actual faint sound between strong auditory stimuli of the bomb falling. Thus, perhaps what participants experienced is simply a conceptual interpretation (i.e., represented but not experienced). Bach (2015) provided a demonstration allowing one to adjust the ISI and stimulus duration. In that video presentation toasting is very apparent at short ISIs, but when the ISI is very long the scene appears to be a series of almost unrelated slices of toasted bread. At those very long ISIs the dynamic process of toasting (and happening) is completely gone.

The exact cognitive mechanism to provide a modal completion of happening is unknown at this time. A mechanism such as orthoscopic perception (Rock, 1997) in which the brain reconstructs the continuous dynamic aspects of scenes from a few discontinuous stimuli is possible but not likely. The participants did not appear to see the walking man between stimuli. Instead, they experienced a nonspecific feeling of action. Regardless of the nature of the stimuli, the dynamic experience was perceived to some extent and was frequency (ISI) dependent. Some may question whether there is any relationship between the phenomenon of happening and phenomenal causality (Hubbard, 2013). However, we do not see any such connection.

\section{Comparison to Other Types of Perceptual Completion}

The modal completion demonstrated in the walking man conditions (at ISI of $0.5 \mathrm{~s}$ ) shares features with that of apparent movement. For example, obstruction (such as the fences in Condition 1) did not prevent the experience from being reported. Research reveals that the course of movement is influenced by the duration of the ISI. Also, Kim, Feldman, and Singh (2012) noted that observers viewed motion sequences of two alternating rectangular tokens positioned at the ends of a semicircular occluder, with varying ISIs (100-500 ms). At short ISIs, observers tended to report simple straight-path motion, that is, outside the occluder. But at long ISIs, they became increasingly likely to report a more appropriate curved-path motion behind the occluder. Similarly, Shiffrar and Freyd (1993) noted that when given enough (ISI) time and appropriate stimuli, the visual system focuses on at least one object-appropriate apparent motion path. Our findings that the walking man seemed to walk 
right through the fence occurred probably because there was no good alternative path for the walking man to take. Additionally, any apparent movement that might have occurred at short ISIs in the walking man condition is more likely to be an example of long-range apparent movement (motion). Shortrange motion system is responsible for performance on a random dot kinematogram. It is thought to occur fairly early in visual processing, before information from the two eyes has been integrated and before shape and color have been extensively analyzed. Long-range motion system is responsible for classic phenomena of apparent motion with large-scale, individual figures. It is thought to occur much later in visual processing, after information from the two eyes has been integrated.

The perceptual weakening with attention shift is not surprising because it is similar to that seen with dynamic change in Nakashima and Yokosawa's (2012) study. Related to attention shift, Muckli, Kohler, Kriegeskorte, and Singer (2005) investigated apparent motion-related activity in patches of $V_{1}$ representing locations along the path of illusory stimulus motion by using functional magnetic resonance imaging. Of interest, that response was unaltered when participants had to perform an attention-demanding task that diverted their attention away from the stimulus.

What is markedly different about the modal completion of happening from other perceptual completions such as apparent movement is that it can be expressed at unusually long ISIs. The experience of modal completion may be not only evoked individually by stimuli but perhaps also evoked more strongly if there is a need for perceptual completion of a scene from a series of stimuli. Nakashima and Yokosawa (2012) did not notice dynamic change when the ISI exceeded 1.0 s. Perhaps that is because some of our stimuli were commonly in the format of a scene (e.g., walking, crawling, and bomb dropping). Zacks and Swallow (2007; see also Radvansky \& Zacks, 2014) noted that brains segment the visual world into scenes.

Our auditory results bring to mind those of Leaver, Van Lare, Zielinski, Halpern, and Rauschecker (2009), who recognized that hearing the end of one album track can elicit a robust image of the upcoming track while one anticipates it in total silence. They referred to it as anticipatory imagery and with the aid of functional magnetic resonance imaging found that anticipatory imagery (in silence) for highly familiar naturalistic music was accompanied by pronounced activity in rostral prefrontal cortex and premotor areas. Whether or not their anticipatory imagery is similar to our experiential phenomenon of happening is speculative.

We do not know, as a result of this study, what the rate of presentation is from merging the separate sequential presentations. That is because widely separated ISIs were tested, none below $0.5 \mathrm{~s}$ and none above $7 \mathrm{~s}$. Our experiments were crude relative to visual flicker fusion and apparent movement experiments. Another potential factor to consider is that of transsaccadic memory. Dickinson and Intraub (2008) described transsaccadic memory in scene perception, which would suggest it might be a factor in the 0.5 -s ISI condition by perceptually completing the interval between stimuli. Although theoretically possible, transsaccadic memory cannot possibly be responsible for happening seen in some participants at 3.0- to 7.0-s ISIs.

\section{Specious Present or "Now"}

The modal completion reported in these experiments was observed with an ISI of $0.5 \mathrm{~s}$ but decreased at $3.0 \mathrm{~s}$ and was seldom reported at 7.0 s. It appears to be no coincidence that the specious present, or now, is found to be about $3.0 \mathrm{~s}$ in duration (Block, 1979; Fairhall, Albi, \& Melcher, 2014; Gruber, 2008; Kinsbourne \& Hicks, 1990; Pöppel, 1997; Ruhnau, 1997). The original estimate of James (1890) was about 6-7 s. It is apparent that there is a connection between now and the modal completion for scenes. Is the perceptual completion of happening a prerequisite for the now, which is, in effect, an experiential phenomenon of vivid memories, dynamic memories, or both? By contrast, memory for events before about $7 \mathrm{~s}$ (outside of the now) does not yield either vivid memories or a dynamic experiential phenomenon. Therefore, perhaps the reason the now is no longer than $3-7 \mathrm{~s}$ is because no individual stimulus can evoke a dynamic perceptual completion (of happening) longer than that.

\section{Summary and Conclusions}

We reported evidence of dynamic perceptual completion as recorded by forced-choice responses to tem- 
porally separated sequences of stimuli in different sensory modalities. The mechanism appears to be that of modal completion in which an illusory percept of happening fills the gap between stimuli. The phenomenon involves dynamic modal completion, and possibly also conceptual processes.

\section{NOTES}

The authors declare that they have no conflicts of interest with respect to their authorship or the publication of this article.

The authors thank Robert Proctor and two anonymous reviewers for helpful comments on this article.

Address correspondence about this article to Richard A. Block, Department of Psychology, Montana State University, Bozeman, MT 59717 (e-mail: block@montana.edu).

\section{REFERENCES}

Bach, M. (2014). Kanizsa triangle [images]. Retrieved from http://www.michaelbach.de/ot/cog_kanizsa/

Bach, M. (2015). The toasting experiment [video]. Retrieved from http://michaelbach.de/ot/o_happening/capp/

Ballard, D. H. (2002). Our perception of the world has to be an illusion. Fournal of Consciousness Studies, 9 , $54-71$.

Blackmore, S. J., Brelstaff, G., Nelson, K., \& Troscianko, T. (1995). Is the richness of our visual world an illusion? Transsaccadic memory for complex scenes. Perception, $24,1075^{-1081 .}$

Block, R. A. (1979). Time and consciousness. In G. Underwood \& R. Stevens (Eds.), Aspects of consciousness: Vol. 1. Psychological issues (pp. 179-217). London, England: Academic Press.

Block, R. A., \& Gruber, R. P. (2014). Time perception, attention and memory: A selective review. Acta Psychologica, 149, 129-133.

Burke, L. (1952). On the tunnel effect. Quarterly fournal of Experimental Psychology, 4, 121-138.

Clark, A. (2002). Is seeing all it seems? Action, reason and the grand illusion. Fournal of Consciousness Studies, 9 , 181-202.

Dickinson, C. S., \& Intraub, H. (2008). Transsaccadic representation of layout: What is the time course of boundary extension? Fournal of Experimental Psychology: Human Perception and Performance, 34, 543-555.

Eagleman, D. M. (2008). Human time perception and its illusions. Current Opinion in Neurobiology, 18, 131-136.

Fairhall, S. L., Albi, A., \& Melcher, D. (2014). Temporal integration windows for naturalistic visual sequences. PLoS ONE, $9(7)$.

Flombaum, J. I., \& Scholl, B. J. (2006). A temporal sameobject advantage in the tunnel effect: Facilitated change detection for persisting objects. Fournal of Experimental
Psychology: Human Perception and Performance, 32, 840-853.

Grondin, S. (2010). Timing and time perception: A review of recent behavioral and neuroscience findings and theoretical directions. Attention, Perception, \& Psychophysics, 72, 561-582.

Gruber, R. P. (2008). Neurophysics of the flow of time. Fournal of Mind and Behavior, 2, 239-254.

Gruber, R. P., Bach, M., \& Block, R. A. (2015). Perceiving two levels of the flow of time. Fournal of Consciousness Studies, 22, 7-22.

Gruber, R. P., \& Block, R. A. (2013). The flow of time as a perceptual illusion. Fournal of Mind and Behavior, 34, 91-100.

Hancock, P. A. (2010). The effect of age and sex on the perception of time in life. American fournal of Psychology, $123,1-13$.

Hancock, P. A. (2011). On the left hand of time. American Fournal of Psychology, 124, 177-188.

Hancock, P. A., \& Block, R. A. (2012). The psychology of time: A view backward and forward. American fournal of Psychology, 125, 267-274.

Hollingworth, A. (2008). Visual memory for natural scenes. In S. J. Luck \& A. Hollingworth (Eds.), Visual memory (pp. 123-162). Oxford, UK: Oxford University Press.

Hubbard, T. L. (2005). Representational momentum and related displacements in spatial memory: A review of the findings. Psychonomic Bulletin \& Review, 1, 822-851.

Hubbard, T. L. (2006). Bridging the gap: Possible roles and contributions of representational Momentum. Psicológica, 27, 1-34.

Hubbard, T. L. (2013). Phenomenal causality I: Varieties and variables. Axiomathes, 23, 1-42.

Hubbard, T. L. (2014). Forms of momentum across space: Representational, operational, and attentional. Psychonomic Bulletin \& Review, 21, 1371-1403.

James, W. (1890). Principles of psychology. New York, NY: H. Holt.

Kellman, P. J., \& Shipley T. F. (1992a). Perceiving objects across gaps in space and time. Current Directions in Psychological Science, 1, 193-199.

Kellman, P. J., \& Shipley, T. F. (1992b). A theory of dynamic occluded and illusory object perception. Fournal of $E x-$ perimental Psychology: General, 135, 513-541.

Kelly, M. H., \& Freyd, J. J. (1987). Explorations of representational momentum. Cognitive Psychology, 19, 369-401.

Keppel, G., \& Wickens, T. D. (2004). Design and analysis: A researcher's handbook (4th ed.). Upper Saddle River, NJ: Pearson.

Kim, S., Feldman, J., \& Singh, M. (2012). Curved apparent motion induced by amodal completion. Attention, Perception, \& Psychophysics, 74, 350-364.

Kinsbourne, M., \& Hicks, R. E. (1990). The extended present: evidence from time estimation by amnesics and nor- 
mals. In V. Giuseppe \& T. Shallice (Eds.), Neuropsychological impairments of short-term memory (pp. 319-330). New York, NY: Cambridge University Press.

Kornmeier, J., \& Bach, M. (2009). Object perception: When our brain is impressed but we do not notice it. Fournal of Vision, 9, 1-10.

Leaver, A. M., Van Lare, J., Zielinski, B., Halpern, A. R., \& Rauschecker, J. (2009). Brain activation during anticipation of sound sequences. Fournal of Neuroscience, 29, $2477-2485$.

$\mathrm{Lg}, \mathrm{V}$. (n.d.). Bomb is falling down from a military aircraft and explode on earth 2. Retrieved from www.pond 5 . com/sound-effect/56414252/bomb-falling-down-andexplode-2.html

Lin, Z., \& He, S. (2012). Emergent filling in induced by motion integration reveals a high-level mechanism in filling in. Psychological Science, 23, 1534-1541.

Macknik, S. L., \& Martinez-Conde, S. (2014). Filling in the Scientific American Mind, 25, 21-23.

Michotte, A., Thines, G., \& Crabbe, G. (1991). Amodal completion of perceptual structures. In G. Thines, A. Costall, \& G. Butterworth (Eds.), Michotte's experimental phenomenology of perception (pp. 140-167). Hillsdale, $\mathrm{NJ}$ : Erlbaum.

Muckli, L., Kohler, A., Kriegeskorte, N., \& Singer, W. (2005) Activity along the apparent-motion trace reflects illusory perception. PLoS Biology, 3(8), e265. doi:10.1371/journal. pbio.0030265

Myers, J. L., Well, A. D., \& Lorch, R. F., Jr. (2010). Research design and statistical analysis. (3rd ed.). New York, NY: Routledge.

Nakashima, R., \& Yokosawa, K. (2012). Sustained attention can create an (illusory) experience of seeing dynamic change. Visual Cognition, 20, 265. [Additional data of a pilot study sent to authors]

Nichols, H. (1891). The psychology of time. American fournal of Psychology, 3, 453-529.

Palmer, E. M., Kellman, P. J., \& Shipley, T. F. (2006). A theory of dynamic occluded and illusory object perception. Fournal of Experimental Psychology: General, 135, 513-541.

Pessoa, L., \& DeWeerd, P. (2003). Filling in: From perceptual completion to cortical reorganization. New York, NY: Oxford University Press.
Pöppel, E. (1997). The brain's way to create "newness." In H. Atmanspacher \& E. Ruhnau (Eds.), Time, temporality, now (pp. 107-120). Berlin, Germany: Springer Verlag.

Radvansky, G. A., \& Zacks, J. M. (2014). Event cognition. New York, NY: Oxford University Press.

Rensink, R. A. (2002). Change detection. Annual Review of Psychology, 53, 249-250.

Rickles, D., \& Kon, M. (Eds.). (2014). Flow of time. In Annals of the New York Academy of Sciences. Malden, MA: Wiley.

Rock, I. (1997). Indirect perception. Cambridge, MA: MIT Press.

Ruhnau, E. (1997). The deconstruction of time and the emergence of temporality. In H. Atmanspacher \& E. Ruhnau (Eds.), Time, temporality, now (pp. 53-70). Berlin, Germany: Springer-Verlag.

Shiffrar, M., \& Freyd, J. J. (1993). Timing and apparent motion path choice with human body photographs. Psychological Science, 4, 379-384.

Simons, D. J., Mitroff, S. R., Franconeri, S. L., \& Peterson, M. A. (2003). Scene perception: What we can learn from visual integration and change detection. In G. Rhodes (Ed.), Perception of faces, objects, and scenes: Analytic and holistic processes (pp. 335-351). New York, NY: Oxford University Press.

Singh, M. (2004). Modal and amodal completion generate different shapes. Psychological Science, 15, 454-459.

VanRullen, R., \& Koch, C. (2003). Is perception discrete or continuous? Trends in Cognitive Sciences, 7, 207-213.

Wokke, M. E., Vandenbroucke, A. R. E., Scholte, H. S., \& Lamme, V. A. F. (2013). Confuse your illusion: Feedback to early visual cortex contributes to perceptual completion. Psychological Science, 24, 63-71.

Yarrow, K., Haggard, P., Heal, R., Brown, P., \& Rothwell, J. C. (2001). Illusory perceptions of space and time preserve cross-saccadic perceptual continuity. Nature, 414 , 302-305.

Zacks, J. M., \& Swallow, K. M. (2007). Event segmentation. Current Directions in Psychological Science, 16, 80-84.

Zakay, D. (2007). Boundaries of psychological time. American fournal of Psychology, 120, 673-677. 